\title{
SOLUTIONS OF FIRST LEVEL OF MEROMORPHIC DIFFERENTIAL EQUATIONS
}

\author{
by W. BALSER
}

(Received 22nd December 1980)

\section{Introduction}

Let a meromorphic differential equation

$$
x^{\prime}=A(z) x, \quad A(z)=z^{r-1} \sum_{s=0}^{\infty} A_{s} z^{-s}
$$

be given, where $r$ is an integer, and the series converges for $|z|$ sufficiently large. Then it is well known that $(0.1)$ is formally satisfied by an expression

$$
H(z)=F(z) z^{L} \exp \{Q(z)\},
$$

where $F(z)$ is a formal power series in $z^{-1}$ times an integer power of $z$, and $F(z)$ has an inverse of the same kind, $L$ is a constant matrix, and

$$
Q(z)=\operatorname{diag}\left[q_{1}(z), \ldots, q_{n}(z)\right]
$$

is a diagonal matrix of polynomials $q_{j}(z)$ in a root of $z, 1 \leqq j \leqq n$. If, for example, all the polynomials in $Q(z)$ are equal, then $F(z)$ can be seen to be a convergent series (see Section 1), whereas if not, then generally the coefficients in $F(z)$ grow so rapidly that $F(z)$ diverges for every (finite) $z$. This roughly indicates that it should be essential to study which of the polynomials are different, and at what "levels" they differ for the first time:

For every pair $j, k$, let $\operatorname{deg}\left(q_{j}(z)-q_{k}(z)\right)$ denote the (rational) exponent of the leading term of $q_{j}(z)-q_{k}(z)$ as $z \rightarrow \infty$; if $q_{j}(z)-q_{k}(z)$ is identically zero, let $\operatorname{deg}\left(q_{j}(z)-q_{k}(z)\right)=-\infty$. Then let $t$ denote the number of different values of $\operatorname{deg}\left(q_{j}(z)-q_{k}(z)\right)$ other than $-\infty$ $(1 \leqq j \leqq n)$. Under various aspects, $t$ indicates how complicated a singularity for the differential equation the point $z=\infty$ is: For example, when calculating the formal solution (or proving its existence), then the calculations are most effectively done in several steps, each of which may be considered to correspond to one of the values of $\operatorname{deg}\left(q_{j}(z)-q_{k}(z)\right)$, hence $t$ indicates how many of these steps are needed. Another example is the proof of the Uniqueness Theorem ([4], pp. 268-272, or [7], pp. 88-96), where $t$ is seen to be the number of different levels in the so-called "iterated block structure", and 
the proof implicitly uses an induction type argument with respect to the levels of this block structure (a different proof is given in [1], [2] where this becomes more explicit).

These two examples support the suggestion that the nature of the singularity of solutions of (0.1) may most naturally be explained in several steps corresponding to the different values of $\operatorname{deg}\left(q_{j}(t)-q_{k}(t)\right)(1 \leqq j, k \leqq n)$. This paper may be visualised as the first step in this direction: We define formal solutions of first level (Section 2) which generalise (0.2) in the sense that instead of $z^{L} \exp \{Q(z)\}$ we allow more general functions $G_{1}(z)$, which however have singularities (at $\left.\infty\right)$ of somewhat simpler nature than those of the solutions of $(0.1)$. These functions $G_{1}(z)$ are fundamental solutions of an "auxiliary" differential equation which we prove to exist in Section 1. In Section 3 we then prove existence and uniqueness of fundamental solutions having a given formal fundamental solution of first level as their asymptotic in sectors of "large" opening and having a Stokes' phenomenon that corresponds to a "block structure of first level". Whenever the parameter $t=1$, our Theorem in Section 3 can be seen to coincide with the Uniqueness Theorem in [4], [7], and for $t>1$, the Uniqueness Theorem could be reproved, using our Theorem, by induction with respect to $t$.

In a separate paper we will show that for formal solutions of first level the coefficients of the formal series $F(z)$ generally grow less rapidly than the coefficients of formal solutions of the usual type. This will be of importance in representing solutions by means of Laplace transformation.

The results of this paper are based upon part of the author's Habilitationsschrift [1].

\section{An auxiliary differential equation}

Throughout this paper, let a fixed, but arbitrary meromorphic differential equation

$$
x^{\prime}=A(z) x
$$

be given, where $A(z)$ is an $n \times n$ matrix of functions that are meromorphic at $\infty$; i.e. for some integer $r$ (the Poincaré rank of (1.1)) and some real $a \geqq 0$ we have

$$
A(z)=z^{r-1} \sum_{s=0}^{\infty} A_{s} z^{-s}, \text { convergent for }|z|>a, A_{0} \neq 0 .
$$

Then (1.1) is known to have a formal fundamental solution $H(z)$ of the form

$$
H(z)=\Psi(z) \exp \{Q(z)\}
$$

with a diagonal matrix $Q(z)$ of polynomials in a root of $z$ without constant terms, and a formal logarithmic matrix $\Psi(z)$ having an inverse of the same type (see [6] for the definition of formal logarithmic matrices and the existence of such formal solutions). In [3], pp. 201-205, the detailed structure of such formal solutions has been investigated; here we only need part of the information upon this structure, which we are going to derive now for the convenience of the reader (for more detailed proofs, however, compare the paper quoted above). 
Since $H\left(z e^{2 \pi i}\right)$ is again a formal fundamental solution of (1.1) (and is even of the same type) we conclude the existence of a constant, invertible matrix written in the form $e^{2 \pi i L}$ such that

$$
H\left(z e^{2 \pi i}\right)=H(z) e^{2 \pi i L}
$$

we refer to $e^{2 \pi i L}$ as the formal monodromy factor of $H(z)$. Using (1.4), we obtain $\exp \{Q(z)\} e^{2 \pi i L} \exp \left\{-Q\left(z e^{2 \pi i}\right)\right\}=\Psi^{-1}(z) \Psi\left(z e^{2 \pi i}\right)$, and since the right-hand side is formally logarithmic, this holds only if the left-hand side is constant; i.e.

$$
Q\left(z e^{2 \pi i}\right)=R^{-1} Q(z) R
$$

for some permutation matrix $R$, and $\mathrm{e}^{2 \pi i L} R^{-1}$ commutes with $\exp \{Q(z)\}$. Therefore, $\Psi\left(z \mathrm{e}^{2 \pi i}\right)=\Psi(z) \mathrm{e}^{2 \pi i L}$, which implies

$$
\Psi(z)=F(z) z^{L}
$$

with a formal meromorphic transformation $F(z)$ (i.e. $F(z)$ is a formal power series in $z^{-1}$ times an integer power of $z$, and $F(z)$ has an inverse of the same kind).

For any polynomial $p(z)$ in a root of $z$, let $\operatorname{deg} p$ (the degree of $p$ ) denote the (rational) exponent of the leading term of $p(z)$ (as $z \rightarrow \infty$ ); if $p(z) \equiv 0$, let $\operatorname{deg} p=-\infty$. Then by $d_{1}$ we denote the maximal degree of the differences of any two of the diagonal elements of $Q(z)$.

If $d_{1}=-\infty$, then all the polynomials are identical (hence according to (1.5) no roots occur), and by a scalar exponential shift $x=\exp \{q(z)\} \tilde{x}$ with a polynomial $q(z)$ we can make the transformed equation $\tilde{x}^{\prime}=\tilde{A}(z) \tilde{x}$ have a regular singularity at $\infty$, which implies that $F(z)$ is convergent. Hence in this case, $H(z)=F(z) z^{L} \exp \{q(z) I\}$ is a proper solution of (1.1) (we use the word proper to distinguish from corresponding formal objects). In order to exclude these trivial cases, we assume:

(a) Let $Q(z)$ contain at least two different polynomials.

Note that assumption (a) always implies $r \geqq 1$, since otherwise $\infty$ would be at most a regular singularity of $(1.1)$, hence $Q(z) \equiv 0$.

In case (a) holds, we may (after possibly rearranging the columns of $H(z))$ assume without loss in generality that those diagonal elements of $Q(z)$ having differences of degree less than $d_{1}$ come consecutively, hence $Q(z)$ may be assumed to have the following structure:

$$
Q(z)=p(z) z^{\left[d_{1}\right]+1} I_{n}+\Lambda z^{d_{1}}+Q^{(1)}(z)
$$

with $p(z)$ being a (possibly identically zero) polynomial in $z,\left[d_{1}\right]$ denotes the largest integer not exceeding $d_{1}, Q^{(1)}(z)$ is a diagonal matrix of polynomials in roots of $z$ (without constant terms) having degrees less than $d_{1}$, and

$$
\Lambda=\operatorname{diag}\left[\lambda_{1} I_{s_{1}}, \ldots, \lambda_{l} I_{s_{l}}\right]
$$


with integer $l \geqq 2$, distinct complex numbers $\lambda_{1}, \ldots, \lambda_{l}$ and natural numbers $s_{1}, \ldots, s_{l}$ (by $I_{s}$ we throughout denote the $s$-dimensional unit matrix for arbitrary natural $s$ ). Analysing (1.5), we see that there exists a unique block permutation matrix $R_{1}$ (in the block structure of $\Lambda$; by block permutation matrix we mean a permutation matrix which, when blocked in a given block structure, has blocks that are either zero or identitymatrices) such that

$$
\Lambda e^{2 \pi i d_{1}}=R_{1}^{-1} \Lambda R_{1}
$$

and the permutation matrix $R R_{1}^{-1}$ is diagonally blocked (in the same block structure). Since $e^{2 \pi i L} R^{-1}$ commutes with $Q(z)$, the matrix $e^{2 \pi i L} R_{1}^{-1}$ necessarily commutes with $\Lambda$ (note that $R R_{1}^{-1}$ commutes with $\Lambda$ anyway), hence

$$
e^{2 \pi i L}=D R_{1},
$$

where $D$ is a constant, invertible, diagonally blocked matrix.

Remark 1.1. Note that the block structure of the matrix $\Lambda$ is the block structure of first level in the iterated block structure which was considered in [1]; [2], pp. 64, 65; [7], pp. 90-92. If one chooses a convenient $a$ priori ordering of the values $\lambda_{1}, \ldots, \lambda_{l}$ (for example lexicographical ordering; [2], p. 63), then the matrix $\Lambda$ and in particular its block structure can be seen to be formally meromorphically invariant. If not otherwise specified, we will throughout this paper take $\lambda_{1}, \ldots, \lambda_{l}$ to be ordered with respect to an arbitrary, but fixed a priori given ordering (so that $\Lambda$ corresponds uniquely to (1.1)), and let all the matrices occurring be blocked in the block structure of first level, i.e. in the block structure of $\Lambda$. If $C=\left[C_{j k}\right]$ is any constant matrix blocked in the block structure of first level, then by $\operatorname{diag}_{1} C$ we denote the diagonally blocked matrix having the form $\operatorname{diag}\left[C_{11}, \ldots, C_{l l}\right]$.

For any fixed $\Lambda$ as in (1.8) and a fixed rational $d_{1}$, we define the dominance relation of first level:

Given a sector $S=S(\alpha, \beta)=\{z ;|z|>R, \alpha<\arg z<\beta\}$, we write

$$
j \prec_{1} k \text { in } S \text { iff } \exp \left\{\left(\lambda_{j}-\lambda_{k}\right) z^{d_{1}}\right\} \rightarrow 0 \text { as } z \rightarrow \infty \text { in } S \text {. }
$$

(Note that we consider the independent variable $z$ on the Riemann surface of the Logarithm so that non-integral powers are always uniquely defined by means of $z^{d_{1}}$ $\left.=\exp \left\{d_{1} \log z\right\}\right)$. The set $\sigma=\sigma(S)$ of pairs $(j, k)$ with $(1.11)$ is then transitive (i.e. $(h, j) \in \sigma$ and $(j, k) \in \sigma$ implies $(h, k) \in \sigma)$ and antisymmetric (i.e., $(j, k) \in \sigma$ contradicts with $(k, j) \in \sigma)$.

The set of constant matrices $C$ for which

$$
\exp \left\{\Lambda z^{d_{1}}\right\} C \exp \left\{-\Lambda z^{d_{1}}\right\} \cong I \text { in } S
$$

shall be denoted by $\mathscr{U}_{1}(S)$. It is immediately clear that $\mathscr{U}_{1}(S)$ is a group with respect to matrix multiplication, and if $C=\left[C_{j k}\right](1 \leqq j, k \leqq l)$ is blocked (with respect to the block 
structure of first level) then $C \in \mathscr{U}_{1}(S)$ iff

$$
C_{j j}=I(1 \leqq j \leqq l), C_{j k}=0 \text { if }(j, k) \notin \sigma(j \neq k, 1 \leqq j, k \leqq l) .
$$

For an arbitrary transitive and antisymmetric set $\sigma$ of pairs $(j, k)$ we define $\mathscr{U}_{1}(\sigma)$ to be the set of constant matrices $C$ satisfying (1.13), and it is easy to see that $\mathscr{U}_{1}(\sigma)$ always is a group. Finally, by $\mathscr{U}_{1}^{+}(S)$ (resp. $\mathscr{U}_{1}^{+}(\sigma)$ for general antisymmetric and transitive $\sigma$ ) we denote the set of invertible constant matrices $C=\left[C_{j k}\right](1 \leqq j, k \leqq l)$ for which

$$
C_{j k}=0 \text { if }(j, k) \notin \sigma(j \neq k, 1 \leqq j, k \leqq l)
$$

(with no restriction upon the diagonal blocks). Since every such transitive and antisymmetric $\sigma$ corresponds to a partial ordering of $\{1, \ldots, l\}$ and can be extended to become a complete ordering, then by a renumeration of the numbers $\lambda_{1}, \ldots, \lambda_{l}$ we may arrange that

$$
(j, k) \in \sigma \text { implies } j<k,
$$

and in this case we see that matrices $C$ satisfying (1.14) are upper triangularly blocked (with possibly additional zero blocks above the diagonal). Hence such a $C$ is invertible iff

$$
\operatorname{diag}_{1} C=\operatorname{diag}\left[C_{11}, \ldots, C_{l l}\right]
$$

is invertible, and $\mathscr{U}_{1}^{+}(\sigma)$ is again a group with respect to matrix multiplication having the property

$$
\operatorname{diag}_{1}\left(C_{1} C_{2}\right)=\operatorname{diag}_{1} C_{1} \operatorname{diag}_{1} C_{2} \text { for } C_{1}, C_{2} \in \mathscr{U}_{1}^{+}(\sigma)
$$

Since a renumeration of the numbers $\lambda_{1}, \ldots, \lambda_{l}$ is reflected in a block-permutationsimilarity of the matrices in $\mathscr{U}_{1}^{+}(\sigma)$, we see that (1.15) and (1.16) generalise to arbitrary numerations of $\lambda_{1}, \ldots, \lambda_{l}$.

Given any equation (1.1) satisfying (a), we are now going to show the existence of an auxiliary equation

$$
\tilde{x}^{\prime}=\tilde{A}(z) \tilde{x}, \tilde{A}(z)=z^{r-1} \sum_{s=0}^{\infty} \tilde{A}_{s} z^{-s}, \tilde{A}_{0} \neq 0
$$

(convergent for $|z|>\tilde{a}$ with suitable $\tilde{a} \geqq 0$ ), which is formally meromorphically equivalent to (1.1), i.e. for some formal meromorphic transformation $T(z)$ we have

$$
\widetilde{A}(z)=T^{-1}(z) A(z) T(z)-T^{-1}(z) T^{\prime}(z),
$$

and in a sense (1.17) has the same Stokes' phenomenon as (1.1), as far as the diagonal blocks of the Stokes' multipliers are concerned, whereas the off-diagonal blocks of the Stokes' multipliers of (1.17) are zero. 
To do so, suppose that for some natural $N$, reals $\alpha_{0}, \ldots, \alpha_{N-1}$ are given with

$$
0 \leqq \alpha_{0}<\alpha_{1}<\cdots<\alpha_{N-1}<2 \pi
$$

and define

$$
\alpha_{-1}=\alpha_{N-1}-2 \pi, \alpha_{N}=\alpha_{0}+2 \pi, \alpha_{N+1}=\alpha_{1}+2 \pi
$$

Then, if we take $N$ and $\alpha_{0}, \ldots, \alpha_{N-1}$ in a way that

$$
\delta=\max \left\{\alpha_{v+1}-\alpha_{v-1} ; 0 \leqq v \leqq N\right\}
$$

is sufficiently small and use a well known Theorem ([10], p. 111), there exist fundamental solutions $Y_{v}(z)$ of (1.1) such that for $v=0, \ldots, N-1$ (with $G(z)=z^{L} \exp \{Q(z)\}$ )

$$
Y_{v}(z) \cong H(z)=F(z) G(z) \text { in } S\left(\alpha_{\nu-1}, \alpha_{v+1}\right)
$$

in the sense that the function $Y_{v}(z) G^{-1}(z)$ has the formal series $F(z)$ as its expansion uniformly in every closed subsector of $S\left(\alpha_{v-1}, \alpha_{v+1}\right)$. Defining

$$
Y_{N}(z)=Y_{0}\left(z e^{-2 \pi i}\right) e^{2 \pi i L}
$$

we conclude, using (1.4), that (1.22) holds also for $v=N$.

Proposition 1. Let any equation (1.1) satisfying (a) be given. Then there exists an equation (1.17), which is formally meromorphically equivalent to (1.1) by means of a suitably fixed transformation

$$
x=T(z) \tilde{x}
$$

such that the following holds:

For any fixed formal fundamental solution $H(z)$ of (1.1) which is of the form (1.3), with $Q(z)$ as in (1.7), define a formal fundamental solution $\tilde{H}(z)$ of $(1.17)$ by

$$
H(z)=T(z) \tilde{H}(z)
$$

Then for every selection of reals $\alpha_{0}, \ldots, \alpha_{N-1}$ as in (1.19) with $\delta$ (as in (1.21)) sufficiently small, and every choice of fundamental solutions $Y_{0}(z), \ldots, Y_{N-1} ; Y_{N}(z)=Y_{0}\left(z e^{-2 \pi i}\right) e^{2 \pi i L}$ of (1.1) which satisfy (1.22) for $v=0, \ldots, N$, there exist fundamental solutions $\widetilde{Y}_{0}(z), \ldots, \widetilde{Y}_{N-1}(z) ; \widetilde{Y}_{N}(z)=\widetilde{Y}_{0}\left(z e^{-2 \pi i}\right) e^{2 \pi i L}$ of (1.17) satisfying

$$
\tilde{Y}_{v}(z) \cong \widetilde{H}(z) \text { in } S\left(\alpha_{v-1}, \alpha_{v+1}\right)(0 \leqq v \leqq N),
$$

such that the matrices $\tilde{W}_{v}=\tilde{Y}_{v}^{-1}(z) \tilde{Y}_{v-1}(z)$ and $W_{v}=Y_{v}^{-1}(z) Y_{v-1}(z)$ are related by

$$
\tilde{W}_{v}=\operatorname{diag}_{1} W_{v}(1 \leqq v \leqq N) \text {. }
$$


Corresponding to a fixed equation (1.1), the auxiliary equation (1.17) is defined up to meromorphic equivalence.

Proof. Suppose that $\alpha_{0}, \ldots, \alpha_{N-1}$ and $Y_{0}, \ldots, Y_{N-1}$ as described above are arbitrarily given (with $\alpha_{-1}, \alpha_{N}, \alpha_{N+1}$, and $Y_{N}$ correspondingly defined), and let (1.24) be regarded as the definition of $\widetilde{W}_{v}(1 \leqq v \leqq N)$. From (1.22) we conclude

$$
G(z) W_{v} G^{-1}(z) \cong I \text { in } S\left(\alpha_{v-1}, \alpha_{v}\right) \quad(1 \leqq v \leqq N)
$$

and since $z^{ \pm L}$ can be estimated by a power of $z$, we find that (1.25) is equivalent to

$$
\exp \{Q(z)\}\left(W_{v}-I\right) \exp \{-Q(z)\} \cong 0 \text { in } S\left(\alpha_{v-1}, \alpha_{v}\right)
$$

for $v=1, \ldots, N$. Since $Q(z)$ is diagonal, (1.26) remains true when replacing $W_{v}$ by $\tilde{W}_{v}$, hence

$$
G(z) \tilde{W}_{v} G^{-1}(z) \cong I \text { in } S\left(\alpha_{v-1}, \alpha_{v}\right) \quad(1 \leqq v \leqq N)
$$

Property (1.27) enables us to apply a Theorem due to Y. Sibuya [8], [9] (see also [4], pp. 280-282) showing the existence of an equation (1.17) having fundamental solutions $\tilde{Y}_{0}(z), \ldots, \widetilde{Y}_{N-1}(z) ; \quad \tilde{Y}_{N}(z)=\tilde{Y}_{0}\left(z e^{-2 \pi i}\right) e^{2 \pi i L}$ satisfying (1.23) (for some formal fundamental solution $\tilde{H}(z)$ of (1.17) of the form $\tilde{H}(z)=\tilde{F}(z) G(z)$ with a formal meromorphic transformation $\tilde{F}(z))$ and $\tilde{Y}_{v-1}(z)=\tilde{Y}_{v}(z) \tilde{W}_{v}(1 \leqq v \leqq N)$, and it is immediate to check that (1.17) is defined up to meromorphic equivalence and is formally meromorphically equivalent to (1.1) by means of $T(z)=F(z) \widetilde{F}^{-1}(z)$.

The constructed equation does not depend upon the choice of $H(z)$, since every other formal fundamental solution of the required form may be written as $H(z) C$ with a constant, invertible $C$ that commutes with $Q(z)$, i.e. is necessarily diagonally blocked. If we correspondingly replace $\tilde{H}(z), Y_{v}(z), \tilde{Y}_{v}(z)$ by $\tilde{H}(z) C, Y_{v}(z) C, \tilde{Y}_{v}(z) C$, resp., then (1.24) remains true (for the matrices $C^{-1} \tilde{W}_{v} C, C^{-1} W_{v} C$ in place of $\tilde{W}_{v}, W_{v}$ ).

To complete the proof, it remains to assure that for different choices of $\alpha_{0}, \ldots, \alpha_{N-1}$, $Y_{0}, \ldots, Y_{N-1}$ we may take the same auxiliary equation (1.17). To do so, assume first that we keep $\alpha_{0}, \ldots, \alpha_{N-1}$ fixed but replace $Y_{0}, \ldots, Y_{N-1}$ by $Y_{0} C_{0}, \ldots, Y_{N-1} C_{N-1}$. Then, in order to make the relation $Y_{N}(z)=Y_{0}\left(z e^{-2 \pi i}\right) e^{2 \pi i L}$ hold for the new matrices as well, we replace $Y_{N}(z)$ by $Y_{N}(z) C_{N}, C_{N}=e^{-2 \pi i L} C_{0} e^{2 \pi i L}$. From (1.22) we conclude that $Y_{v}(z) C_{v} \cong H(z)$ in $S\left(\alpha_{v-1}, \alpha_{v+1}\right)$ iff

$$
\exp \{Q(z)\} C_{\nu} \exp \{-Q(z)\} \cong I \text { in } S\left(\alpha_{\nu-1}, \alpha_{v+1}\right)
$$

for $v=0, \ldots, N-1$ (note that then it automatically follows for $v=N$ according to the definition of $\left.C_{N}\right)$. With $\tilde{C}_{v}=\operatorname{diag}_{1} C_{v}(0 \leqq v \leqq N)$ we conclude as above that (1.28) remains true for $\widetilde{C}_{v}$ instead of $C_{v}$, hence $\tilde{Y}_{v}(z) \tilde{C}_{v} \cong \widetilde{H}(z)$ in $S\left(\alpha_{v-1}, \alpha_{v+1}\right)(0 \leqq v \leqq N$; note that due to (1.10) we have $\tilde{C}_{N}=e^{-2 \pi i L} \tilde{C}_{0} e^{2 \pi i L}$, hence $\left.\tilde{Y}_{N}(z) \tilde{C}_{N}=\tilde{Y}_{0}\left(z e^{-2 \pi i}\right) \tilde{C}_{0} e^{2 \pi i L}\right)$. Using (1.7), we find that $C_{v}, W_{v}, C_{v-1} \in \mathscr{U}_{1}^{+}\left(S\left(\alpha_{v-1}, \alpha_{v}\right)\right)$ for every fixed $v=1, \ldots, N$, hence using (1.16) we 
obtain $\tilde{C}_{v}^{-1} \tilde{W}_{v} \tilde{C}_{v-1}=\operatorname{diag}_{1}\left(C_{v}^{-1} W_{v} C_{v-1}\right)$. This shows that, corresponding to $Y_{0} C_{0}, \ldots, Y_{N-1} C_{N-1}$, the solutions $\tilde{Y}_{0} \tilde{C}_{0}, \ldots, \tilde{Y}_{N-1} \tilde{C}_{N-1}$ have all the properties required in Proposition 1.

To show the independence of the auxiliary equation of the choice of $\alpha_{0}, \ldots, \alpha_{N-1}$, we may restrict to the two cases of either deleting one of these numbers or introducing an additional one; any two choices $\alpha_{0}, \ldots, \alpha_{N-1}$ and $\tilde{\alpha}_{0}, \ldots, \tilde{\alpha}_{N_{-1}}$ may be related by finitely many steps of this "basic" kind. Furthermore, in both cases we may choose particularly convenient solutions $Y_{0}, \ldots, Y_{N-1}$ (since we already know the independence of (1.17) of the choice of solutions). In the first case, assume that we wish to drop the number $\alpha_{\mu}$ with $1 \leqq \mu \leqq N-2$ (similar arguments hold for the remaining cases as well). To do so, there have to exist fundamental solutions of (1.1) having $H(z)$ as their asymptotic in the larger sectors $S\left(\alpha_{\mu-2}, \alpha_{\mu+1}\right)$, resp. $S\left(\alpha_{\mu-1}, \alpha_{\mu+2}\right)$, and if so, we may take these solutions to be $Y_{\mu-1}=Y_{\mu}$, resp. $Y_{\mu+1}$ in the original situation. Then in the new situation, a choice of solutions may be $Y_{0}(z), \ldots, Y_{\mu-1}(z), Y_{\mu+1}(z), \ldots, Y_{N}(z)$, and it is immediate that $\tilde{Y}_{0}(z), \ldots, \widetilde{Y}_{\mu-1}(z), \tilde{Y}_{\mu+1}(z), \ldots, \widetilde{Y}_{N}(z)$ have all the properties desired in the Proposition. In the second case, it is even easier to see how to proceed (in a sense, invert the steps of the first case).

Remark 1.2. If, for example, an equation (1.1) is given with $r \geqq 1$ and $A_{0}$ having all distinct eigenvalues, then

$$
Q(z)=\Lambda_{0} z^{r} / r+Q^{(1)}(z)
$$

where $\Lambda_{0}$ is diagonal and contains the distinct eigenvalues of $A_{0}$, and $Q^{(1)}(z)$ is a diagonal of polynomials in $z$ without constant terms and degrees less than $r$. Hence (1.7) holds with $p(z) \equiv 0, d_{1}=r, \Lambda=\Lambda_{0} / r$. Consequently, the block structure of first level has all one-dimensional blocks. Since it follows from (1.25) that $W_{v}$ always has ones on the diagonal, we see that in this case $\tilde{W}_{v}=I(v=1, \ldots, N)$ follows. A formal solution in this case may be of the form

$$
H(z)=F(z) z^{\wedge^{\prime}} \exp \{Q(z)\}
$$

where $\Lambda^{\prime}$ is diagonal and $F(z)$ is a power series in $z^{-1}$ with leading term $I$. Hence we may take

$$
\widetilde{A}(z)=Q^{\prime}(z)+z^{-1} \Lambda^{\prime}
$$

as an auxiliary equation, and the solution $Y_{v}(z)=z^{\Lambda^{\prime}} \exp \{Q(z)\}(0 \leqq v \leqq N)$, whatever choices for $\alpha_{0}, \ldots, \alpha_{N-1}$ we make.

Another case, in which the auxiliary equation can be taken to be the logarithmic derivative of $G(z)=z^{L} \exp \{Q(z)\}$ is whenever there is only one level in the iterated block structure. In every other case, Proposition 1 proves the existence of an auxiliary equation without saying how to calculate it. Nevertheless, the solutions of the auxiliary equation are simpler than those of the original equation in the sense that they show a Stokes' phenomenon that is diagonally blocked. Using the results of [5], one can show 
that an auxiliary equation is root-meromorphically reducible to a diagonally blocked equation (see also Section 4).

\section{Formal solutions of first level}

For a given equation (1.1) satisfying (a) and a corresponding auxiliary equation (1.17), a formal fundamental solution of first level will be defined to have the form $H_{1}(z)$ $=T(z) G_{1}(z)$, where $T(z)$ is as in Proposition 1 and $G_{1}(z)$ is a fundamental solution of (1.17) having a particularly convenient form. The following Lemma 1 assures the existence of such $G_{1}(z)$ :

Lemma 1. Let an equation (1.1) satisfying (a) be given, and consider any auxiliary equation (1.17) corresponding to (1.1). Then there exists a fundamental solution $G_{1}(z)$ of (1.17) of the form

$$
G_{1}(z)=G^{(1)}(z) \exp \left\{p(z) I_{n} z^{\left[d_{1}\right]+1}+\Lambda z^{d_{1}}\right\}
$$

where $p(z), d_{1}$, and $\Lambda$ are as in (1.7), and $G^{(1)}(z)$ is an invertible matrix such that both $G^{(1)}(z)$ and its inverse are of order smaller than $d_{1}$, i.e. for every sufficiently small $\varepsilon>0$

$$
\exp \left\{-|z|^{d_{1}-\varepsilon}\right\}\left[G^{(1)}(z)\right]^{ \pm 1} \rightarrow 0 \text { as } z \rightarrow \infty \text { in } S(-\infty, \infty)
$$

(uniformly in every closed sector of finite opening).

If $\tilde{G}_{1}(z)=G_{1}(z) C$ for constant, invertible $C$, then $\tilde{G}_{1}(z)$ has the same form as $G_{1}(z)$ iff $C$ is diagonally blocked.

Proof. Using the same notations as in Proposition 1 , define $G_{1}(z)=\widetilde{Y}_{0}(z) C_{0}$ for constant, invertible, diagonally blocked $C_{0}$. Then

$$
G_{1}(z)=\tilde{Y}_{v}(z) C_{v}, C_{v}=\tilde{W}_{v} \ldots \tilde{W}_{1} C_{0}(1 \leqq v \leqq N-1),
$$

and all the $C_{v}$ are diagonally blocked (since the $\tilde{W}_{v}$ are).

Using (1.23), we find

$$
\left\|\left[G_{1}(z) \exp \{-Q(z)\}\right]^{ \pm 1}\right\|=O\left(|z|^{c}\right)\left\|\exp \{Q(z)\} C_{v}^{ \pm 1} \exp \{-Q(z)\}\right\|
$$

in $S\left(\alpha_{v}, \alpha_{v+1}\right)(0 \leqq v \leqq N-1)$ for some real $c>0$. Defining $G^{(1)}(z)$ by (2.1) and using (1.7) together with the fact that $\Lambda$ and $C_{v}$ commute, we have for every sufficiently small $\varepsilon>0$

$$
\left\|\left[G^{(1)}(z)\right]^{ \pm 1}\right\|=o\left(\exp \left\{|z|^{d_{1}-\varepsilon}\right\}\right) \text { as } z \rightarrow \infty \text { in } S\left(\alpha_{-1}, \alpha_{N}\right)
$$

uniformly in every closed subsector. From

$$
\tilde{Y}_{0}(z)=\tilde{Y}_{N}(z) \tilde{W}_{N} \ldots \tilde{W}_{1}=\tilde{Y}_{0}\left(z e^{-2 \pi i}\right) e^{2 \pi i L} \tilde{W}_{N} \ldots \tilde{W}_{1}
$$


we obtain (replacing $z$ by $z e^{2 \pi i}$ and using the definition of $G_{1}(z)$ )

$$
G_{1}\left(z e^{2 \pi i}\right)=G_{1}(z) C_{0}^{-1} e^{2 \pi i L} \tilde{W}_{N} \ldots \tilde{W}_{1} C_{0}
$$

From (1.10) and the fact that $R_{1} \tilde{W}_{N} \ldots \tilde{W}_{1} C_{0} R_{1}^{-1}$ is again diagonally blocked we conclude

$$
G_{1}\left(z e^{2 \pi i}\right)=G_{1}(z) D_{1} R_{1}
$$

with a constant, invertible, diagonally blocked matrix $D_{1}$. Therefore, using (2.1) and (1.9), we find $G^{(1)}\left(z e^{2 \pi i}\right)=G^{(1)}(z) D_{1} R_{1}$, and from (2.3) and the fact that every closed sector of finite opening can be covered by finitely many closed subsectors of the sectors $S\left(\alpha_{-1}+2 k \pi, \alpha_{N}+2 k \pi\right)$ (with arbitrary integer $k$ ), we obtain (2.2).

Now suppose $\tilde{G}_{1}(z)=G_{1}(z) C$ with constant, invertible $C$. Then defining $\widetilde{G}^{(1)}(z)$ analogously to $(2.1)$, we see that $\left[\widetilde{G}^{(1)}(z)\right]^{ \pm 1}$ is of order less than $d_{1}$ iff for every sufficiently small $\varepsilon>0$

$$
\left\|\exp \left\{\Lambda z^{d_{1}}\right\} C^{ \pm 1} \exp \left\{-\Lambda z^{d_{1}}\right\}\right\|=o\left(\exp \left\{|z|^{d_{1}-\varepsilon}\right\}\right)
$$

as $z \rightarrow \infty$ in $S(-\infty, \infty)$, i.e. iff $C$ is diagonally blocked.

Remark 2.1. In the distinct eigenvalue case, i.e. if (1.1) is given with $r \geqq 1$ and $A_{0}$ having all distinct eigenvalues, then one may take $G_{1}(z)=G(z)=z^{\Lambda^{\prime}} \exp \{Q(z)\}$ (compare Remark 1.2), and every other choice, corresponding to the same auxiliary equation, is $G_{1}(z) D$ with a constant, invertible, diagonal $D$. This freedom corresponds precisely to allowing polynomials in $Q(z)$ having non-zero constant terms. We therefore realise that in this simple case there is a natural choice for $G_{1}(z)$ (by taking polynomials without constant terms). In general, it may not be clear how to make a natural choice, however, it will be not essential for our purposes to make any particular choice.

Remark 2.2. Note that it follows from the proof of Lemma 1 that every fundamental solution $G_{1}(z)$ of an auxiliary equation satisfying (2.1), (2.2) has the following property:

For every sector $S$ of sufficiently small opening there exists a diagonally blocked, constant, invertible matrix $C=C(S)$ such that $G_{1}(z) C \exp \{-Q(z)\}$ and the inverse $\exp \{Q(z)\} C^{-1} G_{1}^{-1}(z)$ can be estimated by a power of $|z|$ as $|z| \rightarrow \infty$ in $S$ (uniformly in every closed subsector).

Given an equation (1.1) satisfying (a), we now define a formal fundamental solution of first level to be of the form

$$
H_{1}(z)=T_{1}(z) G_{1}(z)
$$

where $G_{1}(z)$ may be any fundamental solution (of any auxiliary equation $\tilde{x}^{\prime}=\tilde{A}(z) \tilde{x}$ corresponding to (1.1)) which satisfies (2.1), (2.2), and $T_{1}(z)$ is any formal meromorphic transformation which may be taken as $T(z)$ in Proposition 1. 
Note that $H_{1}(z)$ does not determine the factors $T_{1}(z), G_{1}(z)$, since if $T(z)$ is any proper meromorphic transformation, then $H(z)=\tilde{T}_{1}(z) \tilde{G}_{1}(z)$ with $\tilde{T}_{1}(z)=T_{1}(z) T^{-1}(z), \tilde{G}_{1}(z)$ $=T(z) G_{1}(z)$ again satisfies all the conditions (for a generally different auxiliary equation). On the other hand, it appears reasonable to say that whenever in some sense $T_{1}(z) G_{1}(z)$ $=\widetilde{T}_{1}(z) \tilde{G}_{1}(z)$, then $\widetilde{G}_{1}(z) G_{1}^{-1}(z)=\widetilde{T}_{1}^{-1}(z) T_{1}(z)$ (in the sense that $\widetilde{T}_{1}^{-1}(z) T_{1}(z)$ converges for sufficiently large $|z|$ and is the Laurent series representation of $\left.\tilde{G}_{1}(z) G_{1}^{-1}(z)\right)$. To make these arguments precise, we think of a formal fundamental solution of first level to correspond to a pair $T_{1}(z), G_{1}(z)$, and two such pairs $T_{1}(z), G_{1}(z)$ and $\tilde{T}_{1}(z), \tilde{G}_{1}(z)$ are defined to be "equal" (in the sense of giving the same $H_{1}(z)$ ) iff $\widetilde{T}_{1}(z)=T_{1}(z) T^{-1}(z), \widetilde{G}_{1}(z)$ $=T(z) G_{1}(z)$ for some proper meromorphic transformation $T(z)$. We will refer to the pair $T_{1}(z), G_{1}(z)$ as a factorisation of $H(z)$. Choosing a different factorisation can also be visualised as choosing a different auxiliary equation.

Remark 2.3. Let $H_{1}(z)=T_{1}(z) G_{1}(z)$ be a formal fundamental solution of first level of any differential equation (1.1) satisfying (a), and let $e^{2 \pi i L_{1}}$ be its formal monodromy factor, i.e. the unique constant, invertible matrix satisfying

$$
H_{1}\left(z e^{2 \pi i}\right)=H_{1}(z) e^{2 \pi i L_{1}}
$$

Then, since $T_{1}(z)$ is "single valued", i.e. $T_{1}\left(z e^{2 \pi i}\right)=T_{1}(z)$, we have $G_{1}\left(z e^{2 \pi i}\right)=G_{1}(z) e^{2 \pi i L_{1}}$, and from the proof of Lemma 1, especially from (2.4), we conclude

$$
e^{2 \pi i L_{1}}=D_{1} R_{1}
$$

with $R_{1}$ as in (1.9) and $D_{1}$ diagonally blocked, constant, invertible.

Given any formal fundamental solution of first level $H_{1}(z)=T_{1}(z) G_{1}(z)$ of $(1.1)$ and any sector $S$, then a (proper) fundamental solution $X(z)$ of $(1.1)$ is said to have $H_{1}(z)$ as its asymptotic in $S$ (to be asymptotic to $H_{1}(z)$ in $S$ ) iff

$$
X(z) G_{1}^{-1}(z) \cong T_{1}(z) \text { as } z \rightarrow \infty \text { in } S
$$

(uniformly in every closed subsector of $S$ of finite opening), and in this case we write

$$
X(z) \cong H_{1}(z) \text { in } S \text {. }
$$

Note that this definition does not depend upon the particular factorisation of $H_{1}(z)$ into $T_{1}(z), G_{1}(z)$.

Using Proposition 1, we now show that to a given formal fundamental solution of first level, we may always find fundamental solutions having the formal one as their asymptotic in certain sectors and showing a particularly convenient Stokes' phenomenon:

Proposition 2. Let an equation (1.1) satisfying (a) be given, and let $H_{1}(z)=T_{1}(z) G_{1}(z)$ be a fixed, but arbitrary formal fundamental solution of first level of equation (1.1). EMS-F 
Furthermore, let reals $\alpha_{0}, \ldots, \alpha_{N-1}$ as in (1.19) be given and define $\alpha_{-1}, \alpha_{N}, \alpha_{N+1}$ as in (1.20). Then if $\delta$ (as in (1.21)) is sufficiently small, there exist fundamental solutions $Y_{0}(z), \ldots, Y_{N-1}(z) ; Y_{N}(z)=Y_{0}\left(z e^{-2 \pi i}\right) e^{2 \pi i L_{1}}$ (with $e^{2 \pi i L_{1}}$ as in (2.6)) such that

$$
Y_{v}(z) \cong H_{1}(z) \text { in } S\left(\alpha_{v-1}, \alpha_{v+1}\right) \quad(0 \leqq v \leqq N),
$$

and

$$
\operatorname{diag}_{1} W_{v}=I \text {, with } W_{v}=Y_{v}^{-1}(z) Y_{v-1}(z) \quad(1 \leqq v \leqq N)
$$

Remark 2.4. In the distinct eigenvalue case (see Remarks 1.2 and 2.1) we may take $H_{1}(z)=F(z) z^{\Lambda^{\prime}} \exp \{Q(z)\}$ as a formal fundamental solution (of first level as well as in the usual sense), and Proposition 2 is seen to hold trivially since $W_{v}$ automatically has ones on the diagonal. More generally, if in the iterated block structure there is only one level occurring, then again Proposition 2 can be seen to hold trivially. Hence the main case is that two or more levels occur in the iterated block structure.

Proof of Proposition 2. Let $\hat{H}(z)$ be a formal fundamental solution of (1.1) (in the usual sense) that satisfies (1.3), (1.7), and define a formal fundamental solution $\tilde{H}(z)$ of the auxiliary equation $\tilde{x}^{\prime}=\tilde{A}(z) \tilde{x}, \tilde{A}(z)=G_{1}^{\prime}(z) G_{1}^{-1}(z)$, by $\hat{H}(z)=T_{1}(z) \tilde{H}(z)$. Furthermore, let $\hat{Y}_{0}(z), \ldots, \hat{Y}_{N-1}(z) ; \hat{Y}_{N}(z)=\hat{Y}_{0}\left(z e^{-2 \pi i}\right) e^{2 \pi i \hat{L}}$ (with $e^{2 \pi i \bar{L}}$ being the formal monodromy factor of $\hat{H}(z))$ be fundamental solutions of (1.1) with

$$
\hat{Y}_{v}(z) \cong \tilde{H}(z) \text { in } S\left(\alpha_{v-1}, \alpha_{v+1}\right) \quad(0 \leqq v \leqq N)
$$

(which exist if $\delta$ is sufficiently small). Then according to Proposition 1 there exist fundamental solutions $\tilde{Y}_{0}(z), \ldots, \tilde{Y}_{N-1}(z) ; \tilde{Y}_{N}(z)=\tilde{Y}_{0}\left(z e^{-2 \pi i}\right) e^{2 \pi i \tilde{L}}$ of $\tilde{x}^{\prime}=\tilde{A}(z) \tilde{x}$ such that

$$
\tilde{Y}_{v}(z) \cong \tilde{H}(z) \text { in } S\left(\alpha_{v-1}, \alpha_{v+1}\right) \quad(0 \leqq v \leqq N)
$$

and

$$
\tilde{W}_{v}=\tilde{Y}_{v}^{-1}(z) \tilde{Y}_{v-1}(z)=\operatorname{diag}_{1} \hat{W}_{v}, \hat{W}_{v}=\hat{Y}_{v}^{-1}(z) \hat{Y}_{v-1}(z)(1 \leqq v \leqq N)
$$

According to Lemma 1 resp. its proof, we see that $G_{1}(z)=\tilde{Y}_{0}(z) C_{0}, C_{0}$ diagonally blocked, invertible, and therefore

$$
G_{1}(z)=\tilde{Y}_{v}(z) C_{v}, C_{v}=\tilde{W}_{v} C_{v-1} \quad(1 \leqq v \leqq N)
$$

If we define

$$
Y_{v}(z)=\hat{Y}_{v}(z) C_{v} \quad(0 \leqq v \leqq N)
$$

then $Y_{v}(z)$ are fundamental solutions of (1.1) with

$$
Y_{v}(z) G_{1}^{-1}(z)=\hat{Y}_{v}(z) \tilde{Y}_{v}^{-1}(z) \cong T_{1}(z) \text { in } S\left(\alpha_{v-1}, \alpha_{v+1}\right)(0 \leqq v \leqq N),
$$


hence (2.9) follows. Since

$$
Y_{N}(z)=\hat{Y}_{0}\left(z e^{-2 \pi i}\right) e^{2 \pi i \bar{L}} C_{N}=Y_{0}\left(z e^{-2 \pi i}\right) C_{0}^{-1} e^{2 \pi i \bar{L}} C_{N},
$$

and

$$
\begin{aligned}
G_{1}\left(z e^{2 \pi i}\right) & =\tilde{Y}_{N}\left(z e^{2 \pi i}\right) C_{N}=\tilde{Y}_{0}(z) e^{2 \pi i \bar{L}} C_{N} \\
& =G_{1}(z) C_{0}^{-1} e^{2 \pi i \hat{L}} C_{N},
\end{aligned}
$$

we conclude $e^{2 \pi i L_{1}}=C_{0}^{-1} e^{2 \pi i L} C_{N}$, hence

$$
Y_{N}(z)=Y_{0}\left(z e^{-2 \pi i}\right) e^{2 \pi i L_{1}}
$$

Finally, $W_{v}=Y_{v}^{-1}(z) Y_{v-1}(z)=C_{v}^{-1} \hat{W}_{v} C_{v-1}$, and since both $C_{v}$ and $C_{v-1}$ are diagonally blocked and $\tilde{W}_{v}=\operatorname{diag}_{1} \hat{W}_{v}$, we conclude

$$
\operatorname{diag}_{1} W_{v}=C_{v}^{-1} \tilde{W}_{v} C_{v-1}=I \quad(1 \leqq v \leqq N)
$$

Remark 2.5. One can show that the existence of solutions $Y_{0}(z), \ldots, Y_{N}(z)$ as in Proposition 2 is characteristic for formal fundamental solutions of first order:

Let $G_{1}(z)$ be an $n \times n$ matrix analytic for $|z|$ sufficiently large, satisfying $(2.1),(2.2)$ and

$$
G_{1}\left(z e^{2 \pi i}\right)=G_{1}(z) e^{2 \pi i L_{1}}
$$

then it is easy to check that (2.7) holds. If for any choice of reals $\alpha_{0}, \ldots, \alpha_{N-1}$ as in (1.19) with $\alpha_{-1}, \alpha_{N}, \alpha_{N+1}$ as in (1.20), with $\delta$ (as in (1.21)) sufficiently small there exist fundamental solutions $Y_{0}(z), \ldots, Y_{N-1}(z) ; Y_{N}(z)=Y_{0}\left(z e^{-2 \pi i}\right) e^{2 \pi i L_{1}}$ such that

$$
\operatorname{diag}_{1} W_{v}=I, W_{v}=Y_{v}^{-1}(z) Y_{v-1}(z) \quad(1 \leqq v \leqq N)
$$

and for some formal fundamental transformation $T_{1}(z)$

$$
T_{1, v}(z)=Y_{v}(z) G_{1}^{-1}(z) \cong T_{1}(z) \text { in } S\left(\alpha_{v-1}, \alpha_{v+1}\right)(0 \leqq v \leqq N-1) \text {, }
$$

then the function $\tilde{A}(z)=G_{1}^{\prime}(z) G_{1}^{-1}(z)=T_{1, v}^{-1}(z) A(z) T_{1, v}(z)-T_{1, v}^{-1}(z) T_{1, v}^{\prime}(z)$ is single-valued and has $T_{1}^{-1}(z) A(z) T_{1}(z)-T_{1}^{-1}(z) T_{1}^{\prime}(z)$ as its asymptotic expansion in a full neighbourhood of $\infty$, hence $\tilde{x}^{\prime}=\tilde{A}(z) \tilde{x}$ is a meromorphic differential equation and formally meromorphically equivalent to (1.1) by means of $x=T_{1}(z) \tilde{x}$. To show that it is an auxiliary equation corresponding to (1.1), let a formal fundamental solution $\hat{H}(z)$ (in the usual sense) of (1.1) satisfying (1.3), (1.7) be given, and define $\tilde{H}(z)$ by $\hat{H}(z)$ $=T_{1}(z) \tilde{H}(z)$. Then consider fundamental solutions $\hat{Y}_{0}(z), \ldots, \hat{Y}_{N-1}(z) ; \quad \hat{Y}_{N}(z)$ $=\hat{Y}_{0}\left(z e^{-2 \times i}\right) e^{2 \pi i \tilde{L}}$ of $(1.1)$ (with $\left.\hat{H}\left(z e^{2 \pi i}\right)=\hat{H}(z) e^{2 \pi i \tilde{L}}\right)$ satisfying

$$
\hat{Y}_{v}(z) \cong \hat{H}(z) \text { in } S\left(\alpha_{v-1}, \alpha_{v+1}\right) \quad(0 \leqq v \leqq N)
$$


(which exist if $\delta$ is sufficiently small), and define

$$
C_{v}=\hat{Y}_{v}^{-1}(z) Y_{v}(z) \quad(0 \leqq v \leqq N) .
$$

Using (2.1), (2.2), and (1.7) we conclude for sufficiently small $\varepsilon>0$

$$
\exp \left\{\Lambda z^{d_{1}}\right\} C_{\nu} \exp \left\{-\Lambda z^{d_{1}}\right\}=o\left(\exp \left\{|z|^{d_{1}-\varepsilon}\right\}\right)
$$

as $z \rightarrow \infty$ in $S\left(\alpha_{v-1}, \alpha_{v+1}\right)$ which implies $C_{v} \in \mathscr{U}_{1}^{+}\left(S\left(\alpha_{v-1}, \alpha_{v+1}\right)\right)(0 \leqq v \leqq N)$. Therefore $\hat{C}_{v}$ $=C_{v}\left(\operatorname{diag}_{1} C_{v}\right)^{-1} \in \mathscr{U}_{1}\left(S\left(\alpha_{v-1}, \alpha_{v+1}\right)\right)$ which implies

$$
\exp \{Q(z)\} \hat{C}_{v} \exp \{-Q(z)\} \cong I \text { in } S\left(\alpha_{v-1}, \alpha_{v+1}\right) \quad(0 \leqq v \leqq N)
$$

and we may therefore assume that $\hat{C}_{v}=I$ (otherwise, replace $\hat{Y}_{v}(z)$ by $\hat{Y}_{v}(z) \hat{C}_{v}$ which also have $\hat{H}(z)$ as their asymptotic in $S\left(\alpha_{v-1}, \alpha_{v+1}\right)(0 \leqq v \leqq N)$. Hence $C_{v}$ is diagonally blocked, and the solutions $\tilde{Y}_{v}(z)=G_{1}(z) C_{v}^{-1}$ have the properties (1.23) and (1.24) which shows that $\tilde{x}^{\prime}=\tilde{A}(z) \tilde{x}$ is an auxiliary equation (recall from the proof of Proposition 1 that it suffices to consider one choice of $\alpha_{0}, \ldots, \alpha_{N-1}$ and $\left.\hat{Y}_{0}, \ldots, \hat{Y}_{N-1}\right)$, and therefore $H_{1}(z)$ is a formal fundamental solution of first level.

\section{Normal solutions of first level}

In the situation of Proposition 2, we call the solutions $Y_{0}(z), \ldots, Y_{N-1}(z) ; Y_{N}(z)$ $=Y_{0}\left(z e^{-2 \pi i}\right) e^{2 \pi i L_{1}}$ a system of solutions of first level corresponding to $H_{1}(z)$ and $\alpha_{0}, \ldots, \alpha_{N-1}$; the matrices $W_{1}, \ldots, W_{N}$ are named a connection system of first level. Using these notations, Proposition 2 can be thought of as showing the existence of systems of solutions of first level, and we are going to determine the freedom in selecting such a system:

Lemma 2. Let any equation (1.1) satisfying (a) be given, and let $H_{1}(z)=T_{1}(z) G_{1}(z)$ be any formal fundamental solution of first level of equation (1.1).

(a) For any sector $S$ we have $C \in \mathscr{U}_{1}(S)$ iff $\operatorname{diag}_{1} C=I$ and

$$
G_{1}(z) C G_{1}^{-1}(z) \cong I \text { in } S
$$

(uniformly in every closed subsector of $S$ of finite opening).

(b) Corresponding to $H_{1}(z)$ and reals $\alpha_{0}, \ldots, \alpha_{N-1}$, assume the existence of a system of solutions of first level

$$
Y_{0}(z), \ldots, Y_{N-1}(z) ; Y_{N}(z)=Y_{0}\left(z e^{-2 \pi i}\right) e^{2 \pi i L_{1}}
$$

(with $\left.H_{1}\left(z e^{2 \pi i}\right)=H_{1}(z) e^{2 \pi i L_{1}}\right)$. Then for $W_{v}=Y_{v}^{-1}(z) Y_{v-1}(z)$ we have

$$
W_{v} \in \mathscr{U}_{1}\left(S\left(\alpha_{v-1}, \alpha_{v}\right)\right) \quad(1 \leqq v \leqq N),
$$


and $\tilde{Y}_{0}(z), \ldots, \tilde{Y}_{N-1}(z) ; \quad \tilde{Y}_{N}(z)=\tilde{Y}_{0}\left(z e^{-2 \pi i}\right) e^{2 \pi i L_{1}}$ is a system of solutions of first level corresponding to $H_{1}(z)$ and $\alpha_{0}, \ldots, \alpha_{N-1}$ iff the matrices $C_{v}=Y_{v}^{-1}(z) \widetilde{Y}_{v}(z)(0 \leqq v \leqq N)$ satisfy

$$
C_{v} \in \mathscr{U}_{1}\left(S\left(\alpha_{v-1}, \alpha_{v+1}\right)\right) \quad(0 \leqq v \leqq N-1)
$$

and

$$
C_{N}=e^{-2 \pi i L_{1}} C_{0} e^{2 \pi i L_{1}}
$$

(in which case (3.3) follows for $v=N$ as well).

Proof. (a) Using (2.1), (2.2), we obtain for every $C$ with (3.1) (for sufficiently small $\varepsilon>0$ )

$$
\exp \left\{\Lambda z^{d_{1}}\right\} C \exp \left\{-\Lambda z^{d_{1}}\right\}=o\left(\exp \left\{|z|^{d_{1}-\varepsilon}\right\}\right) \text { in } S
$$

hence if $\operatorname{diag}_{1} C=I$, we obtain $C \in \mathscr{U}_{1}(S)$ (compare (1.13)). To prove the converse, we may without loss in generality assume that the opening of $S$ is sufficiently small to apply Remark 2.2 (since otherwise every closed subsector of $S$ with finite opening may be covered by finitely many sectors of arbitrarily small opening). Then according to Remark 2.2 there exists a constant, invertible, diagonally blocked matrix $C_{S}$ such that $G_{1}(z)=F_{S}(z) \exp \{Q(z)\} C_{S}^{-1}$ with an invertible, analytic $F_{S}(z)$ (for $z \in S$ ) such that $F_{S}^{ \pm 1}(z)$ can be estimated by a power of $z$ (as $z \rightarrow \infty$, uniformly in every closed subsector of $S$ ). Hence (3.1) is equivalent to

$$
\exp \{Q(z)\} C_{S}^{-1}(C-I) C_{S} \exp \{-Q(z)\} \cong 0 \text { in } S .
$$

Since $C_{S}$ is diagonally blocked, we see (using (1.13))

$$
C \in \mathscr{U}_{1}(S) \text { iff } C_{S}^{-1} C C_{S} \in \mathscr{U}_{1}(S),
$$

hence if $C \in \mathscr{U}_{1}(S)$, then

$$
\exp \left\{\Lambda z^{d_{1}}\right\} C_{S}^{-1} C C_{S} \exp \left\{-\Lambda z^{d_{1}}\right\} \cong I \text { in } S .
$$

Using (1.7) and $\operatorname{diag}_{1}\left(C_{S}^{-1} C C_{S}\right)=I$, we obtain (3.5), which proves (3.1).

(b) From (2.9) we obtain

$$
G_{1}(z) W_{v} G_{1}^{-1}(z) \cong I \text { in } S\left(\alpha_{v-1}, \alpha_{v}\right) \quad(1 \leqq v \leqq N)
$$

which together with (2.10) implies (3.2) by means of Part (a).

Next, assume $C_{v}$ satisfying (3.3), (3.4) be given, and define $\tilde{Y}_{v}(z)=Y_{v}(z) C_{v}(0 \leqq v \leqq N)$, then $\widetilde{Y}_{N}(z)=\widetilde{Y}_{0}\left(z e^{-2 \pi i}\right) e^{2 \pi i L_{1}}$. Using (a) we obtain

$$
G_{1}(z) C_{v} G_{1}^{-1}(z) \cong I \text { in } S\left(\alpha_{v-1}, \alpha_{v+1}\right)
$$

for $v=0, \ldots, N-1$, and from (3.4) and $G_{1}\left(z e^{2 \pi i}\right)=G_{1}(z) e^{2 \pi i L_{1}}$ we see that (3.6) holds for $v$ $=N$ as well. Furthermore, using (2.7) and the fact that $R_{1}$ is a block permutation 
matrix, we see that $\operatorname{diag}_{1} C_{N}=e^{-2 \pi i L_{1}}\left(\operatorname{diag}_{1} C_{0}\right) e^{2 \pi i L_{1}}=I$, hence $C_{N} \in \mathscr{U}_{1}\left(S\left(\alpha_{N-1}, \alpha_{N+1}\right)\right)$. From (3.6) we conclude $\tilde{Y}_{v}(z) \cong H_{1}(z)$ in $S\left(\alpha_{v-1}, \alpha_{v+1}\right)(0 \leqq v \leqq N)$, and since $\mathscr{U}_{1}\left(S\left(\alpha_{v-1}, \alpha_{v+1}\right)\right)$ and $\mathscr{U}_{1}\left(S\left(\alpha_{v-2}, \alpha_{v}\right)\right)$ are subgroups of the group $\mathscr{U}_{1}\left(S\left(\alpha_{v-1}, \alpha_{v}\right)\right)$, it follows that

$$
\tilde{W}_{v}=\tilde{Y}_{v}^{-1}(z) \tilde{Y}_{v-1}(z)=C_{v}^{-1} W_{v} C_{v-1} \in \mathscr{U}_{1}\left(S\left(\alpha_{v-1}, \alpha_{v}\right)\right)
$$

hence $\operatorname{diag}_{1} \tilde{W}_{v}=I(1 \leqq v \leqq N)$.

Conversely, assume that $\tilde{Y}_{0}(z), \ldots, \tilde{Y}_{N-1}(z) ; \tilde{Y}_{N}(z)=\tilde{Y}_{0}\left(z e^{-2 \pi i}\right) e^{2 \pi i L_{1}}$ is a system of solutions of first level corresponding to $H_{1}(z)$ and $\alpha_{0}, \ldots, \alpha_{N-1}$. Then the matrices $C_{v}$ $=Y_{v}^{-1}(z) \tilde{Y}_{v}(z)$ satisfy (3.4) and (3.6) for $v=0, \ldots, N$. Using (2.1), (2.2) we find for sufficiently small $\varepsilon>0 \exp \left\{\Lambda z^{d_{1}}\right\} C_{v} \exp \left\{-\Lambda z^{d_{1}}\right\}=o\left(\exp \left\{|z|^{\alpha_{1}-\varepsilon}\right\}\right)$ in $S\left(\alpha_{v-1}, \alpha_{v+1}\right)$ which implies $C_{v} \in \mathscr{U}_{1}^{+}\left(S\left(\alpha_{v-1}, \alpha_{v+1}\right)\right)$ (use (1.14)) $(0 \leqq v \leqq N)$. Furthermore, since $C_{v} \tilde{W}_{v}=W_{v} C_{v-1}$ $(1 \leqq \nu \leqq N)$, we conclude

$$
\operatorname{diag}_{1} C_{v}=\operatorname{diag}_{1} C_{v-1} \cdot(1 \leqq v \leqq N)
$$

(note that $C_{v}, \tilde{W}_{v}, W_{v}, C_{v-1}$ are all in $\mathscr{U}_{1}^{+}\left(S\left(\alpha_{v-1}, \alpha_{v}\right)\right.$ ) and use (1.16) together with $\operatorname{diag}_{1} W_{v}=\operatorname{diag}_{1} \tilde{W}_{v}=I$ ). Using $(2.7)$ we see

$$
\operatorname{diag}_{1}\left(e^{-2 \pi i L_{1}} C_{0} e^{2 \pi i L_{1}}\right)=e^{-2 \pi i L_{1}} \operatorname{diag}_{1} C_{0} e^{2 \pi i L_{1}},
$$

hence defining $C=\operatorname{diag}_{1} C_{0}\left(=\operatorname{diag}_{1} C_{v}\right.$ for $\left.v=1, \ldots, N\right)$ we obtain from (3.4)

$$
C=e^{-2 \pi i L_{1}} C e^{2 \pi i L_{1}} \text {. }
$$

Since $C_{v}^{-1} C \in \mathscr{U}_{1}\left(S\left(\alpha_{v-1}, \alpha_{v+1}\right)\right)$, which according to (a) implies $G_{1}(z) C_{v}^{-1} C G_{1}^{-1}(z) \cong I$ in $S\left(\alpha_{v-1}, \alpha_{v+1}\right)$, we conclude from (3.6) that

$$
G_{1}(z) C G_{1}^{-1}(z) \cong I \text { in } \bigcup_{v=0}^{N-1} S\left(\alpha_{v-1}, \alpha_{v+1}\right)=S\left(\alpha_{-1}, \alpha_{N}\right)
$$

Furthermore, (3.7) implies that $G_{1}(z) C G_{1}^{-1}(z)$ is single-valued hence $G_{1}(z) C G_{1}^{-1}(z) \equiv l$, i.e. $C=I$. According to the definition of $C$ this implies (3.3), using (3.6) and (a).

So far, we have considered systems of solutions of first level corresponding to $H_{1}(z)$ and arbitrary reals $\alpha_{0}, \ldots, \alpha_{N-1}$ provided that $\delta=\max \left\{\alpha_{v+1}-\alpha_{v-1} ; 0 \leqq v \leqq N\right\}$ was sufficiently small (to ensure the existence of a system of solutions of first level). We are now going to make a natural selection for these reals.

Let any matrix $\Lambda$ as in (1.8) (with $l \geqq 2$ ) satisfying (1.9) with some fixed rational $d_{1}$ be given. $A$ real number $\tau$ is said to be a first level Stokes' direction if for at least one pair $(j, k), 1 \leqq j, k \leqq l$, and sufficiently small $\varepsilon>0$ we have

$$
j \prec_{1} k \text { in } S(\tau-\varepsilon, \tau) \text {, but } k \prec_{1} j \text { in } S(\tau, \tau+\varepsilon)
$$


(i.e. according to (1.11) the real part of $\left(\lambda_{j}-\lambda_{k}\right) z^{d_{1}}$ changes from negative to positive as $z$ crosses the ray $\arg z=\tau$ in the positive sense). It is clear from the definition that the set of first level Stokes' directions is discrete, hence may be uniquely indexed, using integer indices, such that

$$
\tau_{-1}<0 \leqq \tau_{0}, \tau_{v}<\tau_{v+1} \text { for every integer } v
$$

The set of pairs $(j, k)$ for which (3.8) holds with $\tau=\tau_{v}$ may be denoted by $\rho_{v}$; it is easy to verify that then $\rho_{v}$ is transitive and antisymmetric (for every integer $v$ ).

Let $\mu$ be the number of Stokes' directions in the interval $\left[0, \pi / d_{1}\right)$. It is obvious that for a pair $(j, k)$ the real part of $\left(\lambda_{j}-\lambda_{k}\right) z^{d_{1}}$ changes from negative to positive at $\arg z=\tau$ iff it changes from positive to negative at $\arg z=\tau+\pi / d_{1}$; and

$$
\operatorname{Re}\left(\lambda_{j}-\lambda_{k}\right) z^{d_{1}}>0 \text { for } \tau<\arg z<\tau+\pi / d_{1} \text {. }
$$

Therefore the number of Stokes' directions in any half-open interval of length $\pi / d_{1}$ is equal to $\mu$ (hence $\mu \geqq 1$ ), and we have for every integer $v$

$$
\tau_{v+\mu}=\tau_{v}+\pi / d_{1}, \rho_{v+\mu}=\rho_{v}^{T}=\left\{(j, k) ;(k, j) \in \rho_{v}\right\},
$$

and

$$
\rho_{v}, \rho_{v+1}, \ldots, \rho_{v+2 \mu-1} \text { are pairwise disjoint. }
$$

Remark 3.1. If we define for every $v$

$$
S_{v}=S\left(\tau_{v-1}, \tau_{v+1}\right), \sigma_{v}=\sigma\left(S_{v}\right)
$$

(compare Section 1 for the definition of $\sigma(S)$ ), then

$$
\sigma_{v}^{\prime}=\rho_{v} \cup \cdots \cup \rho_{v+\mu-1}, \sigma_{v}=\sigma_{v}^{\prime} \cap \sigma_{v+1}^{\prime} \text { for every } v
$$

This shows that Lemma 1', (2), p. 68 implies that given matrices $W_{v} \in \mathscr{U}_{1}\left(\sigma_{v}^{\prime}\right)$ (for every $v$ ), there exist unique matrices

$$
C_{v} \in \mathscr{U}_{1}\left(\sigma_{v}\right), V_{v} \in \mathscr{U}_{1}\left(\rho_{v}\right) \text { for every } v
$$

such that

$$
C_{v} V_{v}=W_{v} C_{v-1} \text { for every } v
$$

This will be of importance later.

From (1.9) we conclude the existence of a unique permutation $\Psi$ such that

$$
\lambda_{j} e^{2 x_{i d}}=\lambda_{\Psi(j)}, 1 \leqq j \leqq l,
$$


which shows that if (3.8) holds for a pair $(j, k)$ and a direction $\tau$, then it holds for $(\Psi(j), \Psi(k))$ and $\tau-2 \pi$ as well (and vice versa). Hence if $\tau$ is a Stokes' direction, then $\tau \pm 2 \pi$ is, too, and the number of Stokes' directions in any half-open interval of length $2 \pi$ is the same, say $m$. Therefore

$$
\tau_{v+m}=\tau_{v}+2 \pi \text { for every } v \text {. }
$$

Furthermore, we have for every $v$ (compare (1.13))

$$
C \in \mathscr{U}\left(\rho_{v+m}\right) \text { iff } R_{1}^{-1} C R_{1} \in \mathscr{U}\left(\rho_{v}\right),
$$

or equivalently (since $e^{2 \pi i L_{1}}=D_{1} R_{1}$ with diagonally blocked $D_{1}$, and since $C \in \mathscr{U}\left(\rho_{v}\right)$ iff $\left.D_{1}^{-1} C D_{1} \in \mathscr{U}\left(\rho_{v}\right)\right)$

$$
C \in \mathscr{U}\left(\rho_{\nu+m}\right) \text { iff } e^{-2 \pi i L_{1}} C e^{2 \pi i L_{1}} \in \mathscr{U}\left(\rho_{v}\right)
$$

We are now ready to prove that corresponding to $H_{1}(z)$ and $\tau_{0}, \ldots, \tau_{m-1}$ there exists a system of solutions of first level:

Lemma 3. Let an equation (1.1) satisfying (a) be given, and let $H_{1}(z)=T_{1}(z) G_{1}(z)$ be any fixed, but arbitrary formal fundamental solution of first level of equation (1.1). Then there exist fundamental solutions $Y_{0}(z), \ldots, Y_{m-1}(z) ; Y_{m}(z)=Y_{0}\left(z e^{-2 \pi i}\right) e^{2 \pi i L_{1}}$ of $(1.1)$ with

$$
\begin{gathered}
Y_{v}(z) \cong H_{1}(z) \text { in } S_{v} \quad(0 \leqq v \leqq m), \\
W_{v}=Y_{v}^{-1}(z) Y_{v-1}(z) \in \mathscr{U}_{1}\left(S_{v}^{\prime}\right), S_{v}^{\prime}=S_{v} \cap S_{v-1} \quad(1 \leqq v \leqq m-1) .
\end{gathered}
$$

Proof. Suppose that two sectors $S, \tilde{S}$ with $S \cap \tilde{S} \neq \varnothing$ and two fundamental solutions $X(z), \tilde{X}(z)$ of $(1.1)$ are given with $X(z) \cong H_{1}(z)$ in $S, \tilde{X}(z) \cong H_{1}(z)$ in $\tilde{S}$, and $\operatorname{diag}_{1} W=I$, $W=\tilde{X}^{-1}(z) X(z)$. Then using Lemma 2 (a) we find $W \in \mathscr{U}_{1}(S \cap \tilde{S})$. If $\tilde{S}$ does not contain any Stokes' rays, we have

$$
j{ }_{1} k \text { in } S \cap \tilde{S} \text { implies } j \prec_{1} k \text { in } \tilde{S} \text {, }
$$

hence using (1.13) we conclude

$$
W \in \mathscr{U}_{1}(S \cap \tilde{S}) \text { implies } W \in \mathscr{U}_{1}(\tilde{S})
$$

This proves $X(z)=\tilde{X}(z) W \cong H_{1}(z)$ in $\tilde{S}$, hence

$$
X(z) \cong H_{1}(z) \text { in } S \cup \tilde{S}
$$

Let now (for some natural $N$ ) reals $\alpha_{0}, \ldots, \alpha_{N-1}$ be given, corresponding to which there exists a system

$$
Y_{0}(z), \ldots, Y_{N-1}(z) ; Y_{N}(z)=Y_{0}\left(z e^{-2 \pi i}\right) e^{2 \pi i L 1}
$$


of solutions of first level. According to Proposition 2, the only condition on $\alpha_{0}, \ldots, \alpha_{N-1}$ to ensure the existence of a system of solutions of first level is that $\delta$ as in (1.21) is sufficiently small, hence we may assume that the Stokes' directions in the interval $[0,2 \pi)$ are a subset of $\left\{\alpha_{0}, \ldots, \alpha_{N-1}\right\}$.

For some fixed $v, 0 \leqq v \leqq N-1$, assume that $\alpha_{v}$ is not a Stokes' direction (if no such $v$ exists, then $N=m$ and $\alpha_{v}=\tau_{v}, 0 \leqq v \leqq m$ follows in which case there is nothing to prove). Then the (open) sector $S\left(\alpha_{v-1}, \alpha_{v+1}\right)$ cannot contain any Stokes' ray, and according to the discussion above we have

$$
\begin{gathered}
\left.Y_{v-1}(z) \cong H_{1}(z) \text { in } S\left(\alpha_{v-2}, \alpha_{v+1}\right) \text { (in case } v>0\right), \\
Y_{v+1}(z) \cong H_{1}(z) \text { in } S\left(\alpha_{v-1}, \alpha_{v+2}\right),
\end{gathered}
$$

and therefore

$$
W_{v+1} \in \mathscr{U}_{1}\left(S\left(\alpha_{v-1}, \alpha_{v+1}\right)\right)
$$

Hence if we replace $Y_{v}(z)$ by $Y_{v+1}(z)$ in the system of solutions of first level (in case $v=0$, corresponding replace $Y_{N}(z)$ by $\left.Y_{1}\left(z e^{-2 \pi i}\right) e^{2 \pi i L}\right)$, then either by direct verification or using Lemma 2 we see that the new system is again a system of first level corresponding to $\alpha_{0}, \ldots, \alpha_{N-1}$. Therefore we may assume that $Y_{v}(z)=Y_{v+1}(z)$, and it is immediately checked that removing $\alpha_{v}$ from the collection $\alpha_{0}, \ldots, \alpha_{N-1}$ and correspondingly removing $Y_{v}(z)$ from the system of solutions leads to a system of solutions of first level corresponding to a choice of reals $\alpha_{0}, \ldots, \alpha_{v-1}, \alpha_{v+1}, \ldots, \alpha_{N-1}$ (which still contain the Stokes' directions in $[0,2 \pi))$.

Repeating the above discussion finitely many times finally leads to reals $\alpha_{0}, \ldots, \alpha_{N-1}$ where all $\alpha_{v}$ are Stokes' directions, hence $N=m, \alpha_{v}=\tau_{v}(0 \leqq v \leqq N-1)$ follows, which proves Lemma 3.

Using Lemma 3 we now prove the existence of a unique system of solutions of first level corresponding to $H_{1}(z)$ and $\tau_{0}, \ldots, \tau_{m-1}$ for which the asymptotic holds in sectors of opening larger than $\pi / d_{1}$.

Theorem. Let an equation (1.1) satisfying (a) be given, and let $H_{1}(z)=T_{1}(z) G_{1}(z)$ be any fixed, but arbitrary formal fundamental solution of first level of equation (1.1). Then there is a unique system $X_{0}(z), \ldots, X_{m-1}(z) ; X_{m}(z)=X_{0}\left(z e^{-2 \pi i}\right) e^{2 \pi i L_{1}}$ of solutions of first level corresponding to $H_{1}(z)$ and $\tau_{0}, \ldots, \tau_{m-1}$ characterised by either one of the following two conditions:

$$
X_{v}(z) \cong H_{1}(z) \text { in } S\left(\tau_{v}-\pi / d_{1}, \tau_{v+1}\right) \quad(0 \leqq v \leqq m)
$$

or

$$
V_{v}=X_{v}^{-1}(z) X_{v-1}(z) \in \mathscr{U}_{1}\left(\rho_{v}\right) \quad(1 \leqq v \leqq m)
$$

Proof. Let $Y_{0}(z), \ldots, Y_{m-1}(z) ; Y_{m}(z)=Y_{0}\left(z e^{-2 \pi i}\right) e^{2 \pi i L_{1}}$ be a system of solutions of first level corresponding to $H_{1}(z)$ and $\tau_{0}, \ldots, \tau_{m-1}$ (which exists according to Lemma 3), and 
let $W_{1}, \ldots, W_{m}$ with $W_{v}=Y_{v}^{-1}(z) Y_{v-1}(z)$ be the corresponding connection system. By

$$
W_{v+k m}=e^{-2 k \pi i L_{1}} W_{v} e^{2 k \pi i L_{1}} \text { for } v=1, \ldots, m \text { and integer } k
$$

we define matrices $W_{v}$ which are seen to be in $\mathscr{U}_{1}\left(\sigma_{v}^{\prime}\right)$ for every $v$ (use (1.9), (2.7)). According to Remark 3.1, there exist unique matrices $C_{v} \in \mathscr{U}_{1}\left(\sigma_{v}\right), V_{v} \in \mathscr{U}_{1}\left(\rho_{v}\right)$ (for every v) such that

$$
C_{v} V_{v}=W_{v} C_{v-1} \text { for every } v .
$$

The matrices $\widetilde{V}_{v}=e^{-2 \pi i L_{1}} V_{v-m} e^{2 \pi i L_{1}}$, resp. $\widetilde{C}_{v}=e^{-2 \pi i L_{1}} C_{v-m} e^{2 \pi i L_{1}}$ are also in $\mathscr{U}_{1}\left(\rho_{v}\right)$, resp. $\mathscr{U}_{1}\left(\sigma_{v}\right)$, and since by definition of $W_{v}$ we have $W_{v}=e^{-2 \pi i L_{1}} W_{v-m} e^{2 \pi i L_{1}}$, we find

$$
\tilde{C}_{v} \widetilde{V}_{v}=W_{v} \tilde{C}_{v-1} \text { for every } v \text {. }
$$

Hence from the uniqueness part of Remark 3.1 we conclude

$$
\tilde{C}_{v}=C_{v}, \tilde{V}_{v}=V_{v} \text { for every } v \text {. }
$$

Defining

$$
X_{v}(z)=Y_{v}(z) C_{v} \quad(0 \leqq v \leqq m)
$$

we obtain a system of fundamental solutions which satisfies $X_{m}(z)=Y_{m}(z) C_{m}$ $=Y_{0}\left(z e^{-2 \pi i}\right) e^{2 \pi i L_{1}}\left(e^{-2 \pi i L_{1}} C_{0} e^{2 \pi i L_{1}}\right)=X_{0}\left(z e^{-2 \pi i}\right) e^{2 \pi i L_{1}}$. According to Lemma 2 , $X_{0}(z), \ldots, X_{m-1}(z) ; \quad X_{m}(z)=X_{0}\left(z e^{-2 \pi i}\right) e^{2 \pi i L_{1}}$ is a system of solutions of first level corresponding to $H_{1}(z)$ and $\tau_{0}, \ldots, \tau_{m-1}$, and obviously (3.15) holds. To prove that (3.14) holds as well, note that for $(j, k) \in \rho_{v}$ we have

$$
j \prec_{1} k \text { in } S\left(\tau_{v}-\pi / d_{1}, \tau_{v}\right) \text {, i.e. } V_{v} \in \mathscr{U}_{1}\left(S\left(\tau_{v}-\pi / d_{1}, \tau_{v}\right)\right)
$$

(for every $v$ ). Defining for every integer $k$

$$
X_{v+k m}(z)=X_{v}\left(z e^{-2 k \pi i}\right) e^{2 k \pi i L_{1}} \quad(0 \leqq v \leqq m-1),
$$

we obtain fundamental solutions $X_{v}(z)$ (for every $v$ ) satisfying

$$
X_{v}(z) \cong H_{1}(z) \text { in } S_{v} \text { for every } v .
$$

Suppose that we had shown for some $j, 1 \leqq j \leqq \mu-1$,

$$
X_{v}(z) \cong H_{1}(z) \text { in } S\left(\tau_{v-j}, \tau_{v+1}\right) \text { for every } v
$$

(which is true if $j=1)$, then since $V_{v} \in \mathscr{U}_{1}\left(S\left(\tau_{v}-\pi / d_{1}, \tau_{v}\right)\right.$ ), we conclude (note that $\tau_{v}-\pi / d_{1}$ $\left.=\tau_{v-\mu} \leqq \tau_{v-j-1}\right)$

$$
X_{v}(z)=X_{v-1}(z) V_{v}^{-1} \cong H_{1}(z) \text { in } S\left(\tau_{v-j-1}, \tau_{v}\right) \text { for every } v
$$


which together with $X_{v}(z) \cong H_{1}(z)$ in $S\left(\tau_{v-1}, \tau_{v+1}\right)$ implies

$$
X_{v}(z) \cong H_{1}(z) \text { in } S\left(\tau_{v-j-1}, \tau_{v+1}\right) \text { for every } v .
$$

Hence (3.14) follows by induction.

To prove uniqueness, let $\tilde{X}_{0}(z), \ldots, \tilde{X}_{m-1}(z) ; \tilde{X}_{m}(z)=\tilde{X}_{0}\left(z e^{-2 \pi i}\right) e^{2 \pi i L_{1}}$ be another system of solutions of first level corresponding to $H_{1}(z)$ and $\tau_{0}, \ldots, \tau_{m-1}$ for which

$$
\tilde{X}_{v}(z) \cong H_{1}(z) \text { in } S\left(\tau_{v}-\pi / d_{1}, \tau_{v+1}\right) \quad(0 \leqq v \leqq m)
$$

Then for $C_{v}=X_{v}^{-1}(z) \tilde{X}_{v}(z)$ we obtain

$$
C_{v} \in \mathscr{U}_{1}\left(S\left(\tau_{v}-\pi / d_{1}, \tau_{v+1}\right)\right) \quad(0 \leqq v \leqq m) .
$$

But for every sector $S$ of opening larger than $\pi / d_{1}$ we have $\sigma(S)=\varnothing$, hence $C_{v}=I$ follows, i.e. $\tilde{X}_{v}(z)=X_{v}(z)(0 \leqq v \leqq m)$. Finally, to prove equivalence of $(3.14)$ and (3.15) we are left to show that (3.14) implies (3.15) (the converse has been shown before). This follows since if $X_{v}(z)$ satisfies (3.14) for $v=0, \ldots, m$, then $V_{v}=X_{v}^{-1}(z) X_{v-1}(z) \in \mathscr{U}\left(S\left(\tau_{v}-\pi / d_{1}, \tau_{v}\right)\right)$ for $v=1, \ldots, m$, and $j<_{1} k$ in $S\left(\tau_{v}-\pi / d_{1}, \tau_{v}\right)$ iff $(j, k) \in \rho_{v}$.

Remark 3.2. We call the unique system of solutions of first level satisfying (3.14), (3.15) the system of normal solutions of first level corresponding to $H_{1}(z)$. The corresponding connection system $V_{1}, \ldots, V_{m}$ is called the normalised connection system. Note that the system of normal solutions of first level depends upon the selection of an $a$ priori fixed ordering of the values $\lambda_{1}, \ldots, \lambda_{l}$ in the matrix $\Lambda$, and one can easily see how a change in the ordering of $\lambda_{1}, \ldots, \lambda_{l}$ influences the normal solutions of first level, but we do not want to do this here.

\section{Applications and examples}

\section{(a) Two examples:}

We have seen in Section 3 that the system of normal solutions of first level is uniquely characterised among all systems of solutions of first level by the fact that the asymptotic holds in sectors of the form $S\left(\tau_{v}-\pi / d_{1}, \tau_{v+1}\right)$. In general, this does not imply that (for some fixed $v, 0 \leqq v \leqq m) X_{v}(z)$ is the only fundamental solution having $H_{1}(z)$ as its asymptotic in $S\left(\tau_{v}-\pi / d_{1}, \tau_{v+1}\right)$. To see this, consider an equation (1.1) with

$$
Q(z)=\operatorname{diag}\left[-z, z+z^{1 / 4}, z-z^{1 / 4}, z+i z^{1 / 4}, z-i z^{1 / 4}\right]
$$

(compare [4], pp. 280-282 or [7], pp. 102-108 for the existence of an equation having prescribed invariants). We find

$$
d_{1}=1, \Lambda=\operatorname{diag}\left[-I_{1}, I_{4}\right]
$$


and

$$
\tau_{v}=\pi / 2+v \pi(\text { for every integer } v)
$$

are the Stokes' directions of first level. Then if $H_{1}(z)=T_{1}(z) G_{1}(z)$ is a formal fundamental solution of first level, and $X_{0}(z), X_{1}(z), X_{2}(z)=X_{0}\left(z e^{-2 \pi i}\right) e^{2 \pi i L_{1}}$ are the corresponding normal solutions of first level, we have

$$
Y_{0}(z)=X_{0}(z) C \cong H_{1}(z) \text { in } S(-\pi / 2,3 \pi / 2)
$$

iff

$$
G_{1}(z) C G_{1}^{-1}(z) \cong I \text { in } S(-\pi / 2,3 \pi / 2)
$$

It is easy to see that (4.2) implies

$$
C=\operatorname{diag}\left[I_{1}, \tilde{C}\right]
$$

where $\tilde{C}$ is a $4 \times 4$, invertible matrix, but not necessarily $\tilde{C}=I$ as we will show now:

Using the characterisation of asymptotic sectors ((7), p. 111) we find that every solution of the auxiliary equation, hence in particular $G_{1}(z)$, has a single asymptotic (in the usual sense) in $S(-\pi / 2,3 \pi / 2)$. From the proof of Lemma 1 (also compare Remark 2.2), we see that for some diagonally blocked $D=\operatorname{diag}\left[D_{1}, D_{2}\right]\left(D_{2}\right.$ of size $\left.4 \times 4\right)$ the matrix $G_{1}(z) D \exp \{-Q(z)\}$ and its inverse may be estimated by a power of $z$ as $z \rightarrow \infty$ in $S(-\pi / 2,3 \pi / 2)$ (uniformly in every closed subsector). Hence (4.2) is equivalent to

$$
\exp \{Q(z)\} D^{-1} C D \exp \{-Q(z)\} \cong I \text { in } S(-\pi / 2,3 \pi / 2)
$$

which may be seen to hold for every $C=\operatorname{diag}\left[I_{1}, \tilde{C}\right]$ with

$$
D_{2}^{-1} \tilde{C} D_{2}=\left[\begin{array}{cccc}
1 & 0 & 0 & 0 \\
* & 1 & 0 & * \\
* & 0 & 1 & 0 \\
0 & 0 & 0 & 1
\end{array}\right] \text { (where * denotes an arbitrary entry). }
$$

This shows that for every such $C, Y_{0}(z), X_{1}(z), Y_{2}(z)=Y_{0}\left(z e^{-2 \pi i}\right) e^{2 \pi i L_{1}}$ is a system of solutions of (1.1) satisfying (3.14). This does, however, not contradict the uniqueness part of our Theorem, since for $C \neq I$, this is not a system of first level, since

$$
\operatorname{diag}_{1} X_{1}^{-1}(z) Y_{0}(z)=C \text {. }
$$

Characteristic for the above example is the fact that the Stokes' directions of second level in the iterated block structure ([1]; [2], pp. 64, 65; [7], pp. 90-93) come with a "frequency" $d_{2}=1 / 4$ (i.e. two Stokes' directions to a pair $(j, k)$ come with a minimal distance of $2 \pi / d_{2}=8 \pi$ ) which is much slower than the "frequency" $d_{1}=1$ of the Stokes" 
directions of first level. On the other hand, if the two frequencies are closer together, then one can see that a sector $S\left(\tau_{v}-\pi / d_{1}, \tau_{v+1}\right)$ generally is too large for a solution of (1.1) to have a single asymptotic (in the usual sense) in such a sector: If we take the same example as above, but with $d_{2}=3 / 4$, i.e.

$$
Q(z)=\operatorname{diag}\left[-z, z+z^{3 / 4}, z-z^{3 / 4}, z+i z^{3 / 4}, z-i z^{3 / 4}\right]
$$

then $d_{1}, \Lambda$, and $\tau_{v}$ are as above. A Stokes' direction in the usual sense ([4], p. 262) corresponding to the pair $q_{2}(z)=z+z^{3 / 4}$ and $q_{4}(z)=z+i z^{3 / 4}$ is at $\tau=-\pi / 3$, and a Stokes' direction to the "opposite" pair $q_{4}(z)$ and $q_{2}(z)$ is at $\tau=4 \pi / 3-\pi / 3=\pi$. Since both rays are included in $S(-\pi / 2,3 \pi / 2)$, we have that (in general) no solution of (1.1) may have a single asymptotic (in the usual sense) in the sector $S(-\pi / 2,3 \pi / 2)$ (compare [7], $\mathrm{p}$. 112).

\section{(b) Special factorisations of formal solutions of first level:}

For a fixed formal fundamental solution of first level $H_{1}(z)=T_{1}(z) G_{1}(z)$ the factor $G_{1}(z)$ is defined up to meromorphic equivalence only (see Section 2), and we are going to show that this freedom may be used to arrange for $G_{1}(z)$ to have a particular structure:

In the situation of Proposition 1 we may without loss in generality assume that the auxiliary equation (1.17) is chosen (modulo meromorphic equivalence) such that the formal solution $\tilde{H}(z)$ is of the form

$$
\tilde{H}(z)=\tilde{F}_{b}(z) z^{L} \exp \{Q(z)\}
$$

where $L$ has eigenvalues with real parts in $[0,1)$ (note that $L$ is arbitrary provided $e^{2 \pi i L}$ is the formal monodromy factor hence especially the eigenvalues of $L$ may be changed arbitrarily modulo one) and $\widetilde{F}_{b}(z)$ is a formal power series starting with $I$ (note that, using [3], Lemma 2, every formal meromorphic transformation $\widetilde{F}(z)$ may be factored as $T(z) \tilde{F}_{b}(z)$ with a very simple proper transformation $T(z)$ and $\tilde{F}_{b}(z)$ as above). Defining for $v=0, \ldots, m$

$$
\tilde{\Psi}_{v}(z)=z^{-L} \tilde{Y}_{v}(z) \exp \{-Q(z)\}
$$

we have

$$
\begin{gathered}
z^{L} \tilde{\Psi}_{v}(z) z^{-L} \cong F_{b}(z) \text { in } S\left(\alpha_{v-1}, \alpha_{v+1}\right), \quad 0 \leqq v \leqq m, \\
\tilde{\Psi}_{v-1}(z)=\tilde{\Psi}_{v}(z) \exp \{Q(z)\} \tilde{V}_{v} \exp \{-Q(z)\}, \quad 1 \leqq v \leqq m, \\
\tilde{\Psi}_{m}(z)=e^{-2 \pi i L} \tilde{\Psi}_{0}\left(z e^{-2 \pi i}\right) e^{2 \pi i L}
\end{gathered}
$$

(for (4.8) use $\tilde{Y}_{m}(z)=\tilde{Y}_{0}\left(z e^{-2 \pi i}\right) e^{2 \pi i L}$ and $\left.\exp \{Q(z)\}=e^{-2 \pi i L} \exp \left\{Q\left(z e^{-2 \pi i}\right)\right\} e^{2 \pi i L}\right)$. 
Using our assumption on the eigenvalues of $L$ and computing the explicit form of $z^{J}$, where $J$ is the Jordan canonical form of $L$, one can show that for some real $c, 0<c<1$, we have

$$
\left\|z^{L}\right\|=O\left(|z|^{1-\vartheta}\right),\left\|z^{-L}\right\|=O\left(|z|^{c / 2}\right)
$$

as $z \rightarrow \infty$ in $S\left(\alpha_{v-1}, \alpha_{v+1}\right)$ (for every $\left.v, 0 \leqq v \leqq m\right)$. Since (see (4.6))

$$
z^{L} \tilde{\Psi}_{v}(z) z^{-L}=I+O\left(|z|^{-1}\right) \text { as } z \rightarrow \infty \text { in } S\left(\alpha_{v-1}, \alpha_{v+1}\right)
$$

we have, using these estimates on $z^{ \pm L}$

$$
\tilde{\Psi}_{v}(z) \rightarrow I \text { as } z \rightarrow \infty \text { in } S\left(\alpha_{v-1}, \alpha_{v+1}\right) \quad(0 \leqq v \leqq m) .
$$

Hence the diagonal blocks of $\widetilde{\Psi}_{v}(z)$ are invertible for $|z|$ sufficiently large. Defining

$$
T_{v}(z)=z^{L} \tilde{\Psi}_{v}(z)\left\{\operatorname{diag}_{1} \tilde{\Psi}_{v}\right\}^{-1} z^{-L}
$$

we therefore have that $T_{v}(z)$ is analytic for $|z|$ large, $z \in S\left(\alpha_{v-1}, \alpha_{v+1}\right)$, and using (4.7) and the fact that $\tilde{V}_{v}$ is diagonally blocked, we obtain

$$
T_{v-1}(z)=T_{v}(z)=T(z) \quad(1 \leqq v \leqq m) .
$$

Furthermore, (4.8) and (1.10) imply

$$
\begin{aligned}
T\left(z e^{2 \pi i}\right) & =T_{m}\left(z e^{2 \pi i}\right) \\
& =z^{L} \tilde{\Psi}_{0}(z) D R_{1}\left\{\operatorname{diag}_{1}\left(R_{1}^{-1} D^{-1} \tilde{\Psi}_{0}(z) D R_{1}\right)\right\}^{-1} R_{1}^{-1} D^{-1} z^{-L} \\
& =z^{L} \tilde{\Psi}_{0}(z) D R_{1}\left\{R_{1}^{-1} D^{-1}\left(\operatorname{diag}_{1} \tilde{\Psi}_{0}(z)\right) D R_{1}\right\}^{-1} R_{1}^{-1} \cdot D^{-1} z^{-L} \\
& =T_{0}(z)=T(z)
\end{aligned}
$$

Hence $T(z)$ is single-valued and does not increase faster than some power of $z$ as $z \rightarrow \infty$ in $S\left(\alpha_{-1}, \alpha_{m}\right)$, and so does its inverse. Therefore $T(z)$ is a meromorphic transformation. Applying $T(z)$ to (1.17), we may switch to a (different) auxiliary equation, hence we assume without loss in generality $T(z) \equiv I$. If this is the case, then $\widetilde{Y}_{v}(z)$ is a product of $z^{L}$ times a diagonally blocked matrix $(v=0, \ldots, m)$. Since $G_{1}(z)$ differs from $\tilde{Y}_{v}(z)$ by a constant, diagonally blocked matrix (see the proof of Lemma 1), we see that the same holds for $G_{1}(z)$. This proves, using (2.1), (2.2):

Every formal fundamental solution of first level $H_{1}(z)$ can be written as follows:

$$
H_{1}(z)=T_{1}(z) z^{L} \operatorname{diag}\left[G_{1}^{(1)}(z), \ldots, G_{l}^{(1)}(z)\right]
$$


where $T_{1}(z)$ is a formal meromorphic transformation and

$$
G_{j}^{(1)}(z) \exp \left\{-p(z) z^{\left[d_{1}\right]+1}-\lambda_{j} z^{d_{1}}\right\}
$$

and its inverse is of order smaller than $d_{1}(1 \leqq j \leqq l$.

\section{REFERENCES}

1. W. BALSER, Einige Beiträge zur Invariantentheorie meromorpher Differentialgleichungen (Habilitationsschrift, Ulm, 1978).

2. W. Balser, Zum Einzigkeitssatz in der Invariantentheorie meromorpher Differentialgleichungen, $J$. reine u. angew. Math. 318 (1980), 51-82.

3. W. Balser, W. B. JURKat and D. A. LuTz, A general theory of invariants for meromorphic differential equations. I, formal invariants, Funk. Ekvac. 22 (1979), 197-221.

4. W. Balser, W. B. JuRKat and D. A. LUTz, A general theory of invariants for meromorphic differential equations. II, proper invariants, Funk. Ekvac. 22 (1979), 257-283.

5. W. Balser, W. B. JURKat and D. A. LuTz, The invariants of reducible meromorphic differential equations, Proc. Edinburgh Math. Soc. 23 (1980), 163-187.

6. E. A. Coddington and N. Levinson, Theory of Ordinary Differential Equations (New YorkToronto-London, 1955).

7. W. B. JURKAT, Meromorphe Differentialgleichungen (Lecture Notes in Mathematics 637, Berlin-Heidelberg-New York, 1978).

8. Y. Sibuya, Linear differential equations in the complex domain, Problems of Analytic Continuation (on Japanese) (Kinokuniya, 1976).

9. Y. SibuYa, Stokes' Phenomena, Bull. Amer. Math. Soc. 83 (1977), 1075-1077.

10. W. Wasow, Asymptotic Expansions for Ordinary Differential Equations (New York, 1956).

Abt. Mathematik V

UNIVERSITÄT ULM

D-7900 ULM

West Germany 